\title{
Assessing reliability of myocardial blood flow after motion correction with dynamic PET using a Bayesian framework
}

\author{
Antoine Saillant, Ian Armstrong, Vijay Shah, Sven Zuehlsdorff, \\ Charles Hayden, Jerome Declerck, Kimberley Saint, Matthew Memmott, Mark Jenkinson and Michael Chappell
}

\begin{abstract}
The estimation of myocardial blood flow (MBF) in dynamic PET can be biased by many different processes. A major source of error, particularly in clinical applications, is patient motion. Patient motion, or gross motion, creates displacements between different PET frames as well as between the PET frames and the CT-derived attenuation map, leading to errors in MBF calculation from voxel time series. Motion correction techniques are challenging to evaluate quantitatively and the impact on MBF reliability is not fully understood. Most metrics, such as Signal to Noise Ratio (SNR), are characteristic of static images, and are not specific to motion correction in dynamic data. This study presents a new approach of estimating motion correction quality in dynamic cardiac PET imaging. It relies on calculating a MBF surrogate, $K_{1}$, along with the uncertainty on the parameter. This technique exploits a Bayesian framework, representing the kinetic parameters as a probability distribution, from which uncertainty measures can be extracted. If the uncertainty extracted is high the parameter studied is considered to have high variability - or low confidence - and vice versa. The robustness of the framework is evaluated on simulated time activity curves to ensure that uncertainties are consistently estimated at multiple levels of noise. Our framework is applied on 40 patient datasets, divided in 4 motion magnitude categories. Experienced observers manually realigned clinical datasets with $3 D$ translations to correct for motion. $K_{1}$ uncertainties were compared before and after correction. A reduction of uncertainty after motion correction of up to $60 \%$ demonstrates the benefit of motion correction in dynamic PET and as well as provides evidence of the usefulness of the new method presented.
\end{abstract}

Index Terms-Myocardial Blood flow - Variational Bayes dynamic PET imaging - motion correction - uncertainty measurements.

\section{INTRODUCTION}

$\mathbf{M}$ yocardial Perfusion Imaging (MPI) with hybrid Positron Emission Tomography (PET) / Computed Tomography (CT) is an established technology for assessing coronary artery disease (CAD) [1]. Recent studies have shown that kinetic modelling with dynamic PET provides clinicians with quantitative estimates of Myocardial Blood Flow (MBF), which offers potentially superior diagnosis compared to standard MPI [2], [3], [4]. Quantification and standardization of $\mathrm{MBF}$ is an important area of research for dynamic cardiac PET imaging [5], [6], [7]. By comparing MBF values between a

Copyright (c) 2018 IEEE. Personal use of this material is permitted. However, permission to use this material for any other purposes must be obtained from the IEEE by sending a request to pubs-permissions@ieee.org. resting state and after a response to physiological or pharmacological stress, clinicians can also evaluate Myocardial Flow Reserve (MFR), which is the ratio of stress MBF and rest MBF. Together, MBF and MFR can improve diagnosis, for example in patients with triple vessel coronary disease [8]. However, despite its advantages, the absolute quantification of MBF is not used routinely in the clinic due to a lack of clinical standardization [1], [9]. MBF estimates can be affected by many factors, such as the choice of reconstruction method [10], temporal sampling strategy [11], post-processing methods [6] and patient motion [12].

The impact of reconstruction and acquisition parameters can be minimized with standardization and quality control [1], but patient motion cannot be avoided and is one of the most common issues with dynamic PET in clinical applications, despite careful patient preparation and coaching during the scan. With motion, the region studied might not reflect the same tissue over different image frames, and consequently the final estimate of MBF is less reliable. Moreover, patient motion, or gross motion, is non-periodic, unlike respiratory or cardiac motion. Thus it can be difficult to predict its impact on MBF estimates [13]. Clinical studies, with 90 [13], 225 [14] and 236 patients [15], showed that moderate or high motion can appear relatively frequently, for $30 \%$ to $40 \%$ of the scans, and is more common in stress scans than in rest scans [14]. In addition to frequent occurrence, motion also has a significant impact on MBF estimates. A recent study [12] determined, through computer simulations, that severe patient motion (up to $2 \mathrm{~cm}$ ) could lead to $500 \%$ error in MBF measurement. The impact of motion on clinical data has also been evaluated, demonstrating that motion can be a source of error in MBF estimation [14], [15]. Additionally, motion does not only induce errors within the dynamic PET image but also between the PET series and the CT. A good PET/CT alignment is important for attenuation correction purposes and can be another source of error in the MBF estimation [16].

Motion correction is necessary to perform accurate MBF estimation, but it remains challenging to evaluate quantitatively its accuracy on clinical data, because the ground truth is unavailable. Simulations - with defined ground truth - can be performed in order to establish the performance of motion correction [12], but cannot capture systematically what is happening in actual clinical data. Some groups have evaluated the impact of motion correction on clinical images with generic image quality metrics, such as Signal to Noise Ratio 
(SNR) [17], [18]. Using this approach, an increase of the SNR calculated on the summed image of the dynamic series could demonstrate better image quality, this being associated with success in motion correction [19]. Since this metric is derived from a static image, it cannot characterize the effect of motion correction on dynamic data specifically, which is important for MBF quantification because it relies on features within the dynamic series. Hence a common assessment of the quality of motion correction remains a visual evaluation by a clinical expert [12], [13], which is not objective, being subject to experience and interpretation of the observer. Moreover, these types of metrics do not relate directly with an objective assessment on uncertainty of kinetic parameters and MBF directly. It is recommended that motion correction should be performed before or during reconstruction of the dynamic PET data [6], [12], but currently there is no quantitative way to determine whether motion correction provides more reliable MBF estimates in an individual patient.

To estimate uncertainties of parameters in a model, two different approaches can be used, namely the frequentist and the Bayesian [20]. Despite being often interpreted the same way, some conceptual differences hold. In the frequentist strategy, simulations or clinical studies repeated under the same conditions can be performed to estimate variability or uncertainty in the estimated parameters. By compiling the results of these experiments, statistics can be calculated, from which confidence intervals can be drawn. Yet no inference is possible on the probability that the result of a single experiment lies within the confidence interval. This approach has for example been used to measure the impact of the RenkinCrone parameters on the MBF variability [5]. Nevertheless this technique cannot be used for assessing the variation of a parameter in a single dataset, and hence the effect on parameter variability of a motion correction technique on a particular clinical dataset. The alternative is to appeal to Bayes' theorem, which offers a natural framework to estimate uncertainties of parameters in a model [21]. In a Bayesian framework, each parameter is represented as a probability distribution instead of a single value, from which an uncertainty metric can be drawn. There are various methods to solve the Bayes inference problem [22]. Monte Carlo Markov Chain (MCMC), a family of sampling algorithms, is commonly accepted as the gold standard [23]. However, due to its expensive computations and long processing time [24], it can be impractical to use it in a clinic for kinetic modelling in medical images, especially for voxel-wise analysis [25]. Another way to perform Bayesian inference is to use Variational Bayes (VB) [26], [27], which is a fully Bayesian approach that uses variational theory to approximate the solution to the posterior distribution that is the output of a Bayesian analysis. Thanks to its lower computational cost VB has already been successfully applied to kinetic modelling in medical imaging [28] and also PET kinetic modelling for neurology applications [29].

This study proposes a novel method to assess the motion correction of dynamic cardiac PET data, by evaluating the uncertainty of the MBF estimate, which is derived from the VB framework. Specifically, it is a quantitative way to look at the impact of motion correction on the expected decrease

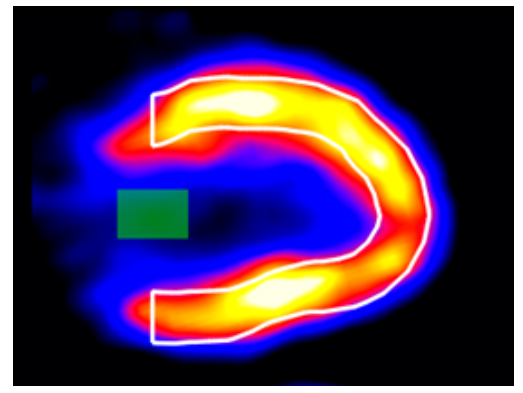

Fig. 1. Central vertical long axis view of the last 5 minutes summed over a 6 minutes dynamic PET cardiac acquisition, started after tracer injection. The green rectangle is the ROI that samples the blood input function in the left ventricle, at the base of the left ventricular cavity. The shape with white contours is the segmentation of the myocardial tissue. The transfer between the blood and the myocardium is modelled by a kinetic model.

of uncertainty after correction. The method is also used to investigate the difference of performing motion correction before or after reconstruction of the PET data. This paper is organized as follows. Section II reviews the theory of PET kinetic modelling and VB inference. Section III provide details on the simulation experiment made to validate the use of VB for measuring MBF uncertainty, as well as the methods applied to compare uncertainties before and after motion correction. Section IV shows the results for the simulations and the clinical datasets. Those results are ultimately discussed in section V.

\section{THEORY}

\section{A. MBF estimation and PET compartmental models}

Computation of MBF requires kinetic modelling of Time Activity Curves (TACs) of the myocardial tissue. TACs represent the evolution of the tracer as a function of time, and can be described by a kinetic model, from which parameters are then subsequently used to compute the MBF.

To compute MBF, TACs are derived from two Regions of Interest (ROIs): the left ventricular (LV) cavity to obtain the arterial blood and the myocardial tissue, as shown in Fig. 1.

The choice of the response function, which describes the residence of the tracer within the tissue, is usually directly inspired by the physiological behavior of the tissue studied [30]. In PET kinetic modeling, compartmental models have been traditionally used for describing the uptake of the tracer in the tissue [31]. Each compartment of the model represents a possible state of the tracer, specifically its physical location or its chemical form. The model depends mainly on the tracer used and the type of the tissue studied. For the tracer Rubidium-82 (Rb-82), used for MBF quantification in this work, the model used is a one-tissue compartment model [30], [32]. The response function $C_{t i s s}(t)$ modeling the tracer exchange from the blood to the tissue is as follows:

$$
C_{\text {tiss }}(t)=K_{1} \exp \left(-k_{2} t\right) \circledast C_{a}(t)
$$

where $C_{a}(t)$ is the time course of the concentration of the tracer in arterial blood also called blood input function; $K_{1}$ 
and $k_{2}$ are the two exchange rates between the blood and the tissue; and $\circledast$ is the convolution operator.

In addition, a partial volume effect correction from the arterial blood is applied [30], as shown in Fig. 2:

$$
C_{m}(t)=\left(1-V_{b}\right) C_{t i s s}(t)+V_{b} C_{a}(t)
$$

where $V_{b}$ is the fraction of volume occupied by arterial blood within the tissue, defined between 0 and $1 ; C_{m}(t)$ is the concentration measured through the dynamic PET series.

Since the blood fraction ratio $V_{b}$ is by definition constrained to the range 0 to 1 , a reparametrization can be used, as is done here:

$$
V_{b}=\frac{1}{\pi} \arctan (x)+\frac{1}{2}
$$

where $x$ is a hyper-parameter, which can take values on $\mathbb{R}$.

In order to obtain the MBF for Rb-82 images, it is necessary to apply the Renkin-Crone equation [32], because of nonlinearity in the relationship between $K_{1}$ and MBF:

$$
K_{1}=\mathrm{MBF} \cdot[1-A \cdot \exp (-B / \mathrm{MBF})]
$$

where: $A$ is defined between 0 and 1 and $B$ is strictly positive. These parameters take different values depending on the tracer properties.

This equation shows that $K_{1}$ is an increasing function of MBF. Therefore a reduction of uncertainty in $K_{1}$ is reflected by a reduction of uncertainty in MBF. Since we are mainly interested in the impact of motion correction on the kinetic parameters for this study, we selected $K_{1}$ as a surrogate for MBF.

\section{B. Measurement of uncertainty with Variational Bayes}

A PET kinetic model is parameterized with a set of $N$ parameters $p=\left\{p_{1} ; \ldots ; p_{N}\right\}$. The measured signal over the $M$ time points is denoted $y=\left\{y_{1} ; \ldots ; y_{M}\right\}$. In our case $N=3$, the parameters are $\left(K_{1} ; k_{2} ; V_{b}\right)$, and $y$ is a time-activity curve (TAC) of the myocardial tissue measured from the PET images. Assuming that the noise on the signal is additive Gaussian noise with precision $\phi$, we define $\Theta=\{p, \phi\}$ as the full set of parameters for the generative model of the data. The PET kinetic model $f(t, p)=C_{m}(t)$, which has been detailed in the previous section (cf. equation 2 ), is used to estimate the most probable kinetic parameters $p$ such that

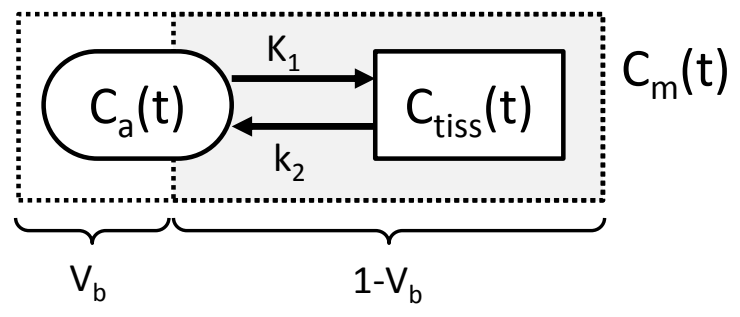

Fig. 2. Representation of a one-tissue compartment model with blood volume fraction and reversible tracer uptake. The MBF is computed from the kinetic parameter $K_{1} . V_{b}$ is the fraction of volume occupied by arterial blood within the tissue. $y=\left\{f\left(t_{j}, p\right)\right\}_{(j \in 1, M)}$.

Using Bayes' theorem we can estimate the posterior probability distribution for the model parameters $\Theta$ given the data $y$ :

$$
P(\Theta \mid y)=\frac{P(y \mid \Theta) P(\Theta)}{P(y)}
$$

Where

- $P(\Theta)$, called prior, is the distribution on the parameters capturing prior knowledge of their value before any new data have been considered, and is often constructed from literature or other data sources;

- $P(y \mid \Theta)$, called the likelihood, is the probability of observing $y$ given a set of parameters $\Theta$, and is computed directly from the model and the observation of the data, i.e. the TAC $y$ in the case of PET kinetics;

- $P(y)$, called the evidence, is the distribution of the observed data, marginalized over the parameters $\Theta, P(y)=$ $\int P(y \mid \Theta) P(\Theta) d \Theta$. The calculation of the evidence $\mathrm{P}(\mathrm{y})$ requires the computation of an integral which is often intractable [24].

MCMC algorithms have been traditionally used to solve the equations arising from Bayes' theorem by sampling the posterior distribution through the construction of a Markov Chain that converges to the posterior distribution after a certain number of iterations. One of the most common MCMC algorithms is the Metropolis-Hastings (MH) method [23]. However those algorithms could be unrealistic to use for the application of medical images in clinical routine, because computing parametric images requires running the fitting algorithm over individual voxels and hence potentially millions of times for a single image, which can take several hours [29].

Variational Bayes resolves this problem by approximating the posterior distribution. VB has been successfully applied to medical imaging thanks to its fast convergence and comparatively inexpensive computations [24], [25]. VB approximates the true posterior $P(\Theta \mid y)$ with a simpler form $Q(\Theta)$. Solving the equations from Bayes' theorem to provide the posterior distribution is then reduced to the maximization of the free energy $F[25]$ :

$$
F=\int Q(\Theta) \log \left(\frac{P(y \mid \Theta) P(\Theta)}{Q(\Theta)}\right) d \Theta
$$

The distribution $Q(\Theta)$ is typically chosen using the mean field approximation; for the kinetic modelling application we consider the parameters of the kinetic model $p$ and the noise model $\phi$ to be independent:

$$
Q(\Theta)=Q_{p}(p \mid y) Q_{\phi}(\phi \mid y)
$$

By using conjugate exponential priors [27], the simplified form $Q(\Theta)$ becomes tractable and the evidence term $P(y)$ can be approximated iteratively. The priors chosen in this work for the application of the VB algorithm in PET kinetic modelling were a multivariate Normal (MVN) for the kinetic model parameters and a Gamma distribution for the noise precision $\phi:$ 


$$
\begin{aligned}
& P(p)=\operatorname{MVN}\left(p, m, \Sigma^{-1}\right) \\
& P(\phi)=\Gamma(\phi, s, c)
\end{aligned}
$$

The interested reader can find the details of the implementation of the VB algorithm here [25], with specifics about the non-linear model iterative optimization. Multiple starting points were used to implement the VB algorithm on a sub grid of values that are positive, and the best fit was kept.

Each kinetic parameter is thus represented by a marginal distribution on $p_{i}, i \in\{1, \ldots, N\}$, which follows a normal distribution $\mathcal{N}\left(\mu_{i} ; \sigma_{i}^{2}\right)$ of respective mean and standard deviation $\left(\mu_{i} ; \sigma_{i}\right)$. The mean $\mu_{i}$ may be taken as the best estimate for the parameter $p_{i}$, while the standard deviation $\sigma_{i}$ is associated with the uncertainty in the measurement. Equally, one can draw credible intervals for the parameters; for example, the 95\% confidence interval $C I_{95}$ of a parameter $p_{i}$ is defined as:

$$
C I_{95}\left(p_{i}\right)=\left[\mu_{i}-1.96 \sigma_{i} ; \mu_{i}+1.96 \sigma_{i}\right]
$$

The wider this interval the more uncertain is the estimated value of $p_{i}$. Another way to look at the uncertainty is to compute the coefficient of variation $C V\left(p_{i}\right)$ :

$$
C V\left(p_{i}\right)=\frac{\sigma_{i}}{\mu_{i}}
$$

The advantage of the coefficient of variation is that it represents a unitless metric that allows comparison across different datasets. Similarly higher values of CV imply higher uncertainty of the parameter $p_{i}$.

\section{Methods AND MATERIALS}

\section{A. Simulations}

1) Data: In order to confirm the validity of the parameter and uncertainty estimates calculated by the Variational Bayes algorithm on PET kinetic models, we used simulated time activity curves (TACs) with known ground truth (Table I). Those parameters were compared to a Metropolis-Hastings (MH) implementation of the MCMC algorithm, as it is regarded as a reference method in the literature. The values chosen for $K_{1}, k_{2}$ and $V_{b}$ correspond to typical values of stress and rest [32].

TABLE I

VALUES OF KINETIC PARAMETERS USED FOR SIMULATIONS.

\begin{tabular}{|c|c|c|c|}
\hline & $\begin{array}{c}K_{1} \\
(\mathrm{~mL} / \mathrm{min} / \mathrm{g})\end{array}$ & $\begin{array}{c}k_{2} \\
(1 / \mathrm{min})\end{array}$ & $\begin{array}{c}V_{b} \\
\text { (unitless) }\end{array}$ \\
\hline TAC-1 (Rest) & 0.47 & 0.12 & 0.48 \\
\hline TAC-2 (Stress) & 1.08 & 0.21 & 0.50 \\
\hline
\end{tabular}

The TACs generated were a sequence of time points, associated with a given activity, representing the mid-frame time of the frame. The activity at a given point was taken as the mean activity integrated over the time duration of the frame. The frames had the following duration: $1 \times 10$ seconds, $8 \times 5$ seconds, $3 \times 10$ seconds, $2 \times 20$ seconds and $4 \times 60$ seconds, which is the framing strategy used in a clinical workflow for PET cardiac studies [10] and for the clinical data used in this study. The blood input function (BIF), corresponding to $C_{a}(t)$ in equation (1), was extracted from clinical data, using a commercial PET cardiology software (syngo.PET Myocardial Blood Flow - Siemens Medical Solutions USA, Inc., Malvern, PA, USA) [33]. Noise was added to the TACs in order to simulate realistic data. The variance of the Gaussian noise was approximated following recommendations from previous studies [34], [35]:

$$
\sigma_{\phi}^{2}=\alpha^{2} \frac{y\left(t_{i}\right)}{\Delta t_{i}}
$$

Where $y\left(t_{i}\right)$ is the simulated noise free TAC at frame $i$ in $\mathrm{Bq} / \mathrm{mL}$, of mid-time $t_{i}$ in seconds, $\Delta t_{i}$ is the $\mathrm{i}$ th frame length and $\alpha$ is a constant determining the noise level. For each noise level 1000 realisations were generated. $\alpha$ was varied to have 15 values: $\{10,20,30,40,50,60,70,80,90,100,110,120,130,140,150\}$ and each noise level is denoted $N L=\{1, \ldots, 15\}$. NL values between 10 and 15 correspond to typical values for polar map TACs in Rb-82 dynamic PET. The purpose of simulations was to obtain TACs that are similar to what can be encountered in our clinical data. Since the clinical datasets were reconstructed iteratively with an OSEM method [36], a non-negativity constraint was added to the simulated TACs. Potential noise realizations that generated negative activities were discarded and regenerated until a positive value was obtained. Since the clinical data were decay corrected, the decay of the tracer was not included in our noise modeling.

2) Analysis: To evaluate the reliability of the mean estimate $\hat{\mu}_{K_{1}}$, the relative error $E r r$ for each realization of each noise level was calculated as follows:

$$
E r r=\frac{\widehat{\mu}_{K_{1}}-K_{1_{O}}}{K_{1_{O}}}
$$

where: $K_{1_{O}}$ is the ground truth value of $K_{1}$

To evaluate the reliability of the uncertainty estimate $\widehat{\sigma}_{K_{1}}$ from the VB algorithm, 95\% confidence intervals $\widehat{C I}_{V B}\left(K_{1}\right)$ on the $K_{1}$ distribution were used. Those intervals were calculated for each TAC at each noise level. In order to estimate the reliability of those confidence intervals, a frequentist approach was used: we counted for each noise realization of a given noise level, the number of times $K_{1_{O}}$, the ground truth value of $K_{1}$, fell into the estimated confidence intervals $\widehat{C I}_{V B}\left(K_{1}\right)$. This frequency is denoted $f_{\widehat{C I}_{V B}}$ :

$$
f_{\widehat{C I}_{V B}}\left(K_{1}\right)=\frac{1}{n} \sum_{j=1}^{n} \delta^{j}
$$

Where:

$$
\delta^{j}=\left\{\begin{array}{ll}
1 & \text { if } K_{1_{O}} \in \widehat{C I}_{V B}^{j}\left(K_{1}\right) \\
0 & \text { if } K_{1_{O}} \notin \widehat{C I}_{V B}^{j}\left(K_{1}\right)
\end{array} ;\right.
$$

$j$ is the $\mathrm{j}$-th random noise realization of a given noise level;

$$
\widehat{C I}_{V B}^{j}\left(K_{1}\right)=\left[\widehat{\mu}_{K_{1}}^{j}-1.96 \widehat{\sigma}_{K_{1}}^{j} ; \widehat{\mu}_{K_{1}}^{j}+1.96 \widehat{\sigma}_{K_{1}}^{j}\right] ;
$$


$n$ is the number of noise realizations and $\left(\widehat{\mu}_{K_{1}}^{j}, \widehat{\sigma}_{K_{1}}^{j}\right)$ are the respective estimated mean and standard deviation of the $K_{1}$ density distribution for the $\mathrm{j}$-th TAC of a given noise level.

If the uncertainty estimate from the VB method is acceptable then the value $f_{\widehat{C I}_{V B}}$ should be constant across the different noise levels. As the noise increases, $\widehat{\sigma}_{K_{1}}$ will increase as well, but the proportion of true values within the confidence interval should remain the same. If the value of $f_{\widehat{C I}_{V B}}$ is close to $95 \%$, the Bayesian definition of the confidence interval is in agreement with the frequentist definition.

The same method was then applied to confidence intervals drawn from an implementation of the Metropolis-Hastings algorithm (CI) $\widehat{C I}_{M H}\left(K_{1}\right)$. The Metropolis-Hastings chain was run with 15,000 iterations with the first 5,000 discarded as burn-in. The same prior distributions were used as for VB. The frequency that the ground truth fell into the estimated confidence intervals $\widehat{C I}_{M H}\left(K_{1}\right)$ was calculated:

$$
f_{\widehat{C I}_{M H}}\left(K_{1}\right)=\frac{1}{n} \sum_{j=1}^{n} \delta^{j}
$$

Where:

$$
\delta^{j}=\left\{\begin{array}{ll}
1 & \text { if } K_{1_{O}} \in \widehat{C I}_{M H}^{j}\left(K_{1}\right) \\
0 & \text { if } K_{1_{O}} \notin \widehat{C I}_{M H}^{j}\left(K_{1}\right)
\end{array} ;\right.
$$

$\mathrm{j}$ is the $\mathrm{j}$-th random noise realization of a given noise level; and $\mathrm{n}$ is the number of noise realizations.

The $95 \%$ confidence interval $\widehat{C I}_{M H}^{j}$ was directly defined from the posterior distribution sampled from the Metropolis Hastings algorithm.

Finally, the metric used to compare the correct estimation of $\hat{\sigma}_{K_{1}}$ was the difference of each frequency calculated:

$$
\epsilon=f_{\widehat{C I}_{V B}}-f_{\widehat{C I}_{M H}}
$$

\section{B. Clinical Data}

1) Acquisition and reconstruction: 40 datasets from a patient cohort, referred for clinically indicated $\mathrm{Rb}-82$ dynamic PET/CT, were analyzed. Data were completely anonymised before inclusion, collected as part of routine clinical management and retrospectively analysed. Datasets were not taken consecutively, as the purpose of the study was to obtain 10 datasets in each motion group. The demographics of the patients were as follows: 25 males and 15 females, 61.3 years old on average ( \pm 11.5 years) and an average weight of 94.5 $\mathrm{kgs}( \pm 23.0 \mathrm{kgs})$. Advice from the local ethics committee deemed that the use of retrospective anonymised patient data did not require formal ethical approval.

The patient data were categorized in 4 different groups based on the amount of motion, according to the method in [13], with 10 datasets in each group. Group 0 was characterized by no motion, while group 1 contained light motion (less than half the width of the left ventricular (LV) myocardial wall), group 2 contained moderate motion (greater than half the width of the LV myocardial wall but could be corrected

\section{Step 1:}

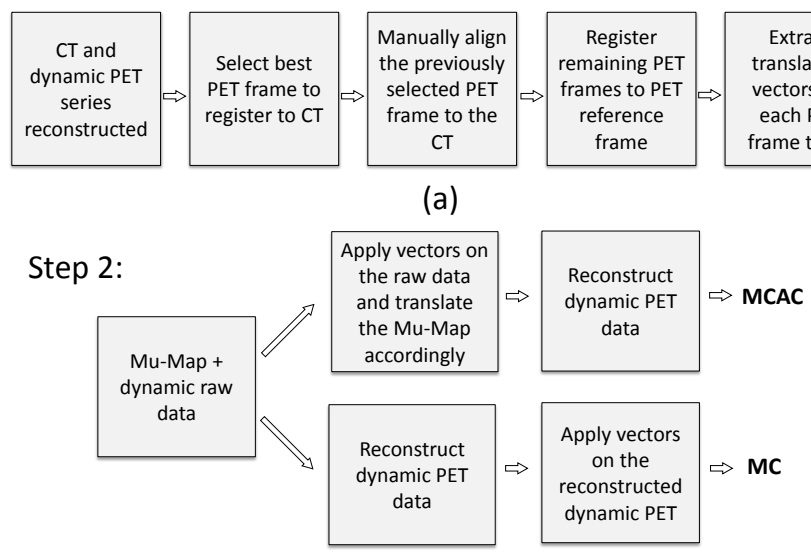

(b)

Fig. 3. Motion alignment workflow strategy.

a) Step 1: Workflow used for finding motion vectors of each PET frame. b) Step 2: Difference of motion alignment strategy: either before or after the reconstruction

partially by the automated motion correction algorithm of syngo.PET MBF) and group 3 contained severe motion cases (same as 2 but could not be corrected by the automated motion correction algorithm of syngo.PET MBF, visually assessed by review of the dynamic PET series post-correction). Only stress images were considered for this study, since motion is more frequent and pronounced in stress patients than rest. Patients were administered with $1110 \mathrm{MBq}(30 \mathrm{mCi})$ of $\mathrm{Rb}-$ 82, generated from a Cardiogen 82-Rb generator (Bracco Diagnostics, Milan, Italy), which is the dose recommended by a previous study [37] to avoid any detector saturation. Additionally, no evidence of scanner detector saturation was found post-acquisition. The stress agent used was adenosine, with a dose of $140 \mu \mathrm{g} / \mathrm{kg} / \mathrm{min}$. Images were acquired on a Biograph mCT (Siemens Medical Solutions USA, Inc., Knoxville, TN, USA). The data were collected for 6 minutes after injection of the tracer. Only one CT was acquired for both Stress and Rest protocol. The time sampling used for the reconstruction was: $1 \times 10$ seconds, $8 \times 5$ seconds, $3 \times 10$ seconds, 2x20 seconds and 4x60 seconds (identical to the simulations). Each dynamic frame was reconstructed with a 3D Ordered Subset Expectation Maximization (OSEM) [36] using 2 iterations, 24 subsets and a $6.5 \mathrm{~mm}$ full-width half maximum Gaussian post-filter. In addition, each frame was decay corrected.

2) PET frames realignment workflow : Frames were manually realigned by three experienced observers in nuclear cardiology. The alignment process consisted of two steps, Fig. 3. This process is referred as motion correction in this study.

The first step consisted of manually estimating the motion, see Fig. 3a: For each dataset, one of the four last frames of the PET series was manually aligned to the CT image and acted as a reference. For a given subject, every observer used the same reference PET frame to align with the CT image. Once this reference PET frame was aligned with the CT, all other PET frames were manually aligned to reference PET frame. Three 
dimensional translations were used to correct for motion.

The second step consisted of applying the motion realignment vectors from step 1, see Fig. 3b. For the purposes of this study this was done in two different ways:

1) A dynamic series was created by applying the motion vectors to the raw data. The attenuation correction map, or Mu-Map, which is a tissue density map, generated from the estimated density of the tissue from the CT image, was shifted for each frame, in order to keep the $\mathrm{CT}$ and the PET frame aligned. By shifting the $\mathrm{Mu}-$ Map, the attenuation correction was specific for each frame. This series is referred as Motion Corrected with Attenuation Correction (MCAC).

2) An additional dynamic series was created from the original series, by reconstructing the dynamic series and then applying the motion vectors defined by observers. This series is referred as Motion Corrected (MC).

$\mathrm{MC}$ and MCAC images had the same motion realignment vectors; the only difference was the stage in the reconstruction in which the motion realignment was applied. Consequently, the two images differed in intensity, due to the different reconstruction methods.

3) Time-activity curves extraction: Time-activity curves were obtained with the syngo.PET MBF software application [38]. The default automatic motion correction was disabled for the processing of each of the three series Original, MC and MCAC. This software package automatically segments the left ventricle and generates a polar map which is a $2 \mathrm{D}$ representation of the $3 \mathrm{D}$ segmentation of the myocardium. The arterial blood input function ROI and the myocardium segmentation were defined automatically by syngo.PET MBF. In order to minimize the effect of partial volume, the base and apex definition were manually adjusted from the initial segmentation, if found to be necessary. Similarly the long axis could be re-angulated if required. The position of the long axis was chosen to decrease the spillover between the blood pool and the myocardium segmentation as much as possible. Additionally the same long axis was chosen for processing MCAC and MC data, so that a given TAC described the same region of interest for both corrected series.

Syngo.PET MBF application generates 540 TACs (36 points defined on the maximum line from the center at regular angles for each 15 different slices). The TACs were regrouped into 4 categories: 3 coronary territories (left anterior descendingLAD, left circumflex-LCX, right coronary artery-RCA) and the whole myocardium (Total). Those 3 coronary territories were the ones defined by syngo.PET MBF software as shown in Fig. 4.

4) Analysis: The effectiveness and reliability of a motion correction strategy was evaluated by computing the normalized difference of the estimated uncertainty of $K_{1}$ :

$$
\Delta \sigma_{K_{1}}=\frac{\sigma_{K_{1_{C}}}-\sigma_{K_{1_{O}}}}{\sigma_{K_{1_{O}}}}
$$

where: $\sigma_{K_{1} C}$ is the uncertainty on $K_{1}$ after correction (realignment of PET frames) and $\sigma_{K_{1} O}$ is the uncertainty on $K_{1}$ before correction. If $\Delta \sigma_{K_{1}}<0$ the correction has reduced

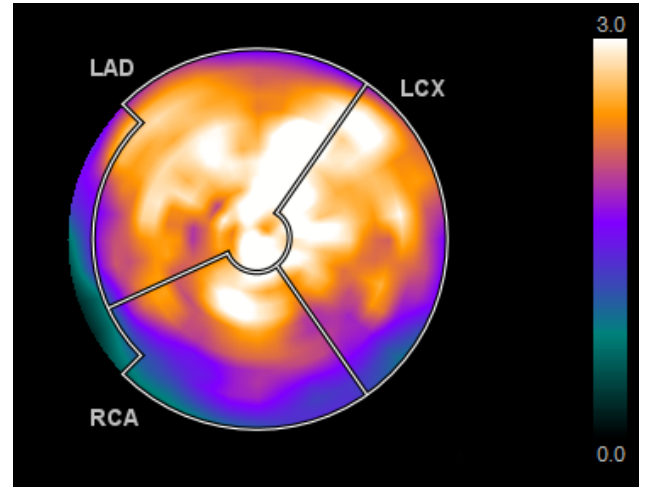

Fig. 4. Example of a MBF polar map generated by syngo.PET MBF from a dynamic PET acquisition. The three different territories (LAD, LCX and RCA) can be seen. Values of the polar map are typical $\mathrm{MBF}$ values in $\mathrm{mL} / \mathrm{g} / \mathrm{min}$ for a normal stress scan.

the uncertainty of $K_{1}$, i.e. motion correction resulted in a higher confidence in $K_{1}$. A negative change means that the motion corrected image provides a more reliable estimate of $K_{1}$ than the original series. A paired t-test was performed in order to evaluate the statistical difference between $\sigma_{K_{1} C}$ and $\sigma_{K_{1} O}$. To compare the effectiveness of the MC method over the MCAC, the standard deviation $\sigma_{K_{1}}$ (see equation 3 ) and the mean value $\mu_{K_{1}}$ of each dataset were computed for each observer. Similarly a paired t-test was computed between $\sigma_{K_{1}{ }_{M C}}$ and $\sigma_{K_{1}{ }_{M C A C}}$ as well as between $\mu_{K_{1}{ }_{M C}}$ and $\mu_{K_{1}{ }_{M C A C}}$, where the subscript indicates the respective MC and MCAC corrections described in section III-B2. Finally, to evaluate the effectiveness of motion correction in comparison to motion free datasets, the coefficient of variation $C V_{K_{1}}$ was computed for each dataset after correction and compared to group 0 datasets.

\section{RESULTS}

\section{A. Simulation}

Fig. 5 shows representative simulated noisy rest TACs at two different noise levels. As expected, the noisier curve leads to a wider $\widehat{K_{1}}$ distribution.

The relative error in the mean estimate $\widehat{\mu}_{K_{1}}$ was robust to different noise levels, as shown in Fig. 6. The average error for the noise levels 5,10 and 15 were respectively of $-0.3 \%,-0.9 \%$ and $-2.1 \%$ for the Rest TAC, and $0.09 \%,-0.2 \%$ and $-0.7 \%$ for the Stress TAC. Fig. 7 shows the quantities $f_{\widehat{C I}_{V B}}$ and $f_{\widehat{C I}_{M H}}$, representing the frequency with which the ground truth value of $K_{1}$ fell into the $95 \%$ confidence interval estimated by the different inference approaches. For the Rest TAC, both methods gave reliable estimates of the $95 \%$ confidence intervals (respectively $\widehat{C I}_{M H}$ for $\mathrm{MH}$ and $\widehat{C I}_{V B}$ for $\mathrm{VB}$ ); the values were on average $94.6 \%$ for $\mathrm{VB}$ and $94.5 \%$ for $\mathrm{MH}$ algorithm. For the Stress TAC, both methods evaluated the confidence intervals with a small difference compared to the frequentist definition, with $f_{\widehat{C I}_{V B}}$ and $f_{\widehat{C I}_{M H}}$ being respectively $89.5 \%$ and $88.1 \%$. The difference, $\epsilon$, between $f_{\widehat{C I}_{V B}}$ and $f_{\widehat{C I}_{M H}}$ was small for the Rest TAC with an average difference of $0.08 \%$. Similar findings were observed for the Stress TAC with an average difference of $1.2 \%$. For both TACs 

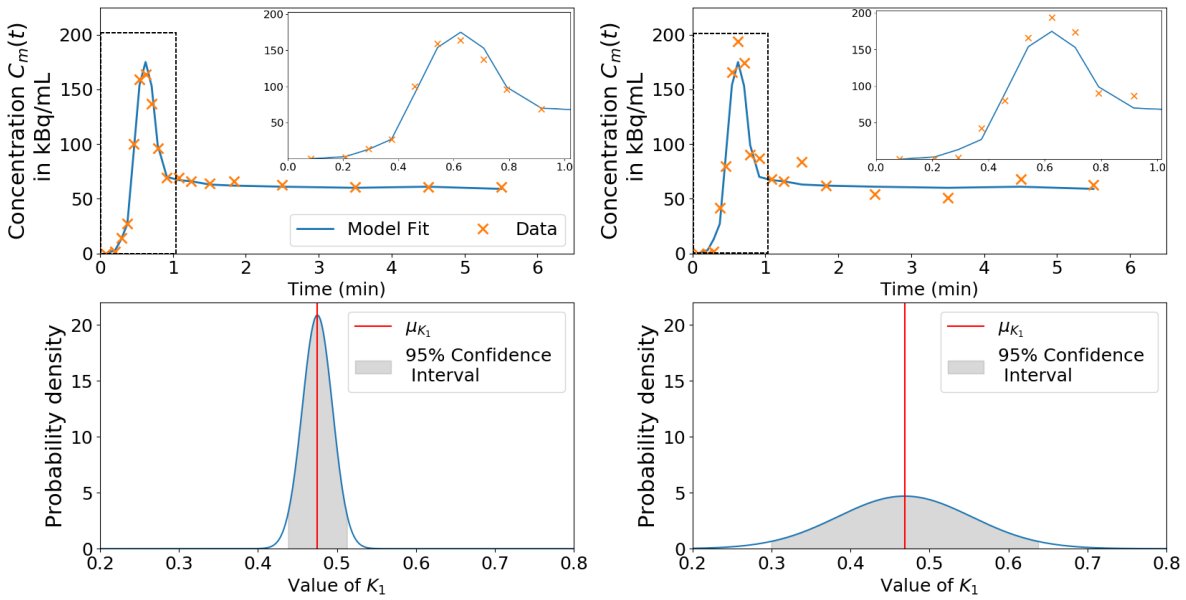

Fig. 5. Examples of model fitting using VB. The top row shows two graphs representing simulated Rest TACs with different levels of noise (respectively 5 and 15). Noise level of 15 is what is typically seen in clinical data. For each graph of the top row, the inset plot shows the data for the first minute of the acquisition. The bottom row shows the impact on the estimated distribution of the parameter $K_{1}$. One can see that the graph on the bottom left, has a narrower distribution than the graph on the bottom right $\left(\widehat{\sigma}_{K_{1}}=0.019 \mathrm{~mL} / \mathrm{min} / \mathrm{g}\right.$ for noise level $=5$ and $\widehat{\sigma}_{K_{1}}=0.084 \mathrm{~mL} / \mathrm{min} / \mathrm{g}$ for noise level $=$ $15)$. Hence the noisier curve leads to higher uncertainty and less reliability.
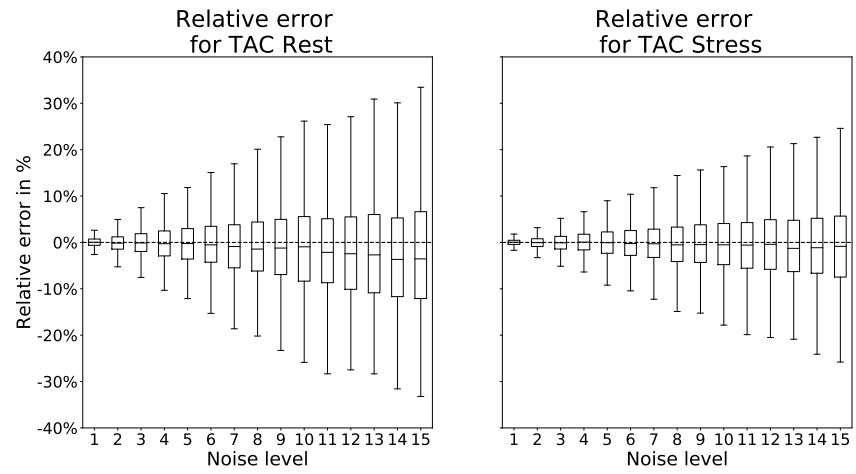

Fig. 6. Relative errors for estimating K1 both TAC Rest and Stress. The mean estimate $\widehat{\mu}_{K_{1}}$ shows robustness to different noise levels. The box plot represents the distribution of the realizations for a given noise level. The median is represented by the middle of the box, the first and third quartiles by the edges, and first and last decile by the whiskers.

the results did not vary substantially across the different noise levels, as shown in Fig. 7.

\section{B. Clinical data}

The ranges of motion vectors applied for frames realignment are detailed in Table II.

Overall a reduction in $\Delta \sigma_{K_{1}}$ after MCAC correction for the data in the whole myocardium was observed in the three motion groups, Fig. 8. For motion group 3, the mean reduction for the whole myocardium was $45.3 \%$ on average for the three observers. The biggest change was seen for the RCA with a reduction in uncertainty of $63.6 \%$. Similar results were observed for motion group 2 - a reduction of $40.5 \%$ was observed on average for the whole myocardium for the three observers. The RCA territory showed the largest decrease at $56.0 \%$ on average. As expected, because there was less motion
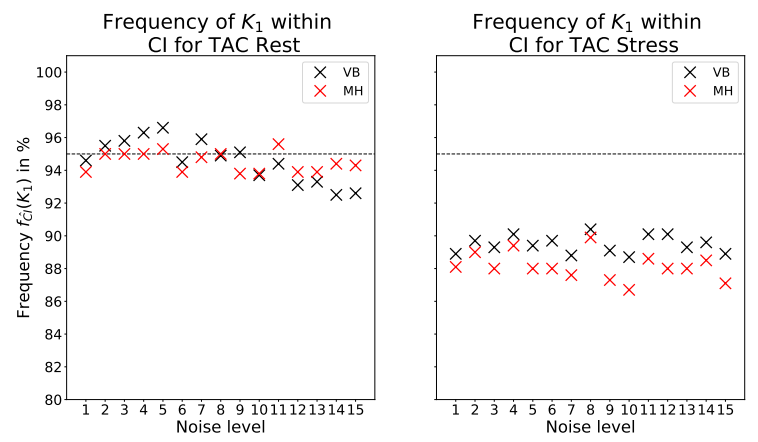

Fig. 7. Comparison between $f_{\widehat{C I}}$ and $f_{\widehat{C I}}$ for the different noise level and each simulated TAC (Stress and Rest). The dashed line in the graphs shows the $95 \%$ value. The difference was small for both TACs, showing that the $95 \%$ confidence interval was estimated with similar performance for the VB algorithm and for $\mathrm{MH}$.

TABLE II

RANGE OF MOTION VECTORS FOR OBSERVER 1 FOR EVERY DATASET OF EACH MOTION GROUP

\begin{tabular}{|c|c|c|c|}
\hline & $\begin{array}{c}\text { Light motion } \\
\text { (group 1) }\end{array}$ & $\begin{array}{c}\text { Moderate motion } \\
\text { (group 2) }\end{array}$ & $\begin{array}{c}\text { Severe motion } \\
\text { (group 3) }\end{array}$ \\
\hline $\begin{array}{c}\text { Range for } \\
\text { average } \\
\text { translation (mm.) }\end{array}$ & {$[0.8 ; 2.1]$} & {$[1.4 ; 5.7]$} & {$[3.3 ; 8.2]$} \\
\hline
\end{tabular}

The ranges given are for relative frame realignment (i.e frame-to-frame). The norm of the translation used to correct for relative realignment is calculated for each frame and averaged across the number of PET frames. Average relative alignments are computed for each dataset in each motion category. The intervals indicate the minimum and maximum values in a motion category. Ranges correlate with the qualitative motion categorization of the datasets.

than in groups 2 and 3, changes were smaller for group 1, but the territory most affected remained the RCA with a mean reduction of $10.1 \%$. For every observer, statistical differences between $\sigma_{K_{1_{M C A C}}}$ and $\sigma_{K_{1_{O}}}$ were found (p-value $\left.<0.05\right)$ for 
both moderate and severe motion in the whole myocardium and the RCA.

The distribution of $\sigma_{K_{1 M C}}$ and $\sigma_{K_{1 M C A C}}$ were found to be very similar, as shown in Fig. 9. For the 3 motion groups, the average values were indistinguishable for the whole myocardium ( $p$-value $>0.05$ ). Similar conclusions were observed when comparing $\mu_{K_{1}}$ after MC and MCAC, (p-value $>0.05$ ).

Fig. 10 details the values of $C V_{K_{1}}$ for observer 1 before and after MCAC. As expected, before correction $C V_{K_{1}}$ was largest in groups with greatest motion. The distribution of $C V_{K_{1}}$ values after MCAC was very similar across all motion groups, with for example mean values for the total territory of $10.3 \%$ in group 0 and $8.0 \%$ for group $1,11.5 \%$ for group 2 and $10.4 \%$ for group 3. Similar results were found for individual territories. While there were some individual differences for a given dataset between the three observers (data not shown), no major differences were found in $C V_{K_{1}}$ between the three observers. Similar observations made about Fig. 8 can be done about the change of $C V_{K_{1}}$ after motion correction: the uncertainty reduced most for high and moderate motion, while being more modest for light motion.

\section{DISCUSSION}

In this work, a method to evaluate the reliability of motion correction techniques through a Bayesian framework has been developed and presented. To estimate kinetic parameters of dynamic PET alongside with an uncertainty measurement, the Variational Bayes framework has been chosen due to its fast convergence and relatively inexpensive computations.

Our method was initially evaluated using simulations. The uncertainties estimates of the kinetic parameters were found to be well estimated at all noise levels: that is, while the uncertainty increased progressively with an increase in noise, as shown in Fig. 5, the proportion of simulations where the true value fell within the confidence interval were similar from one noise level to another. The estimates from VB were very close to the confidence intervals derived from an $\mathrm{MH}$ implementation, which is normally regarded as the "gold standard" for Bayesian inference, albeit usually computationally impractical for routine application to parametric estimation in medical images. To evaluate the validity of the uncertainty measures arrived at by Bayesian inference we compared the predictions from VB and $\mathrm{MH}$ to the ensemble confidence interval from Monte Carlo simulation, i.e. a frequentist measure. For the simulated Rest case there was a close agreement between Bayesian and frequentist definitions of the confidence intervals, with values of $f_{\widehat{C I}_{V B}}$ and $f_{\widehat{C I}_{M H} \text {. }}$ close to $95 \%$. For the Stress case both Bayesian methods similarly estimate the confidence interval. However the values of $f_{\widehat{C I}_{V B}}$ and $f_{\widehat{C I}_{M H}}$ were around $90 \%$, showing a small difference between frequentist and Bayesian definitions of the confidence intervals. Given the agreement between VB and MH this difference is unlikely to be as a result of the approximations made in the VB algorithm. However, it may well be reflective of the nature of the non-linear kinetic model employed resulting in a deviation between the confidence interval as might be defined using a Bayesian or frequentists approach. In the context of this work, this does not imply that the use of uncertainty would be unreasonable for use as a way to assess improvements in pre-processing, such as motion correction. The robustness of the estimates $\left(\widehat{\mu}_{K_{1}} ; \widehat{\sigma}_{K_{1}}\right)$ (Fig. 7) is of particular importance for the application to motion correction, because motion can be seen as another source of noise, albeit potentially more complex in practice than the Gaussian noise assumption made in the simulations.

Our implementation of the $\mathrm{MH}$ algorithm processed 1000 TACs in 42 minutes and 20 seconds. The VB algorithm processed the same 1000 TACs in 6 seconds. Those results were obtained with an Intel Xeon CPU E5-1620 of 3.70 $\mathrm{GHz}$ and with $16.0 \mathrm{~GB}$ of RAM. An implementation of the VB algorithm can be found in the FMRIB Software library (www.fmrib.ox.ac.uk/fsl) [25], which is freely available for academic use.

The uncertainty metric was applied to clinical data in order to assess the effectiveness of manual motion correction carried out by expert clinical observers. This was done first by comparing the values of $\sigma_{K_{1}}$ (Fig. 8), before and after motion correction. A significant drop in $\sigma_{K_{1}}$ was observed for moderate and high motion for both the total myocardial tissue and the RCA territory, with a trend toward reduction in the other territories. Values of $C V_{K_{1}}$ after motion correction were also compared to $C V_{K_{1}}$ before correction and those values found from motion-free datasets (Fig. 10). The results showed that the level of uncertainties after motion correction were similar between each motion group and reduced to a comparable level to the data judged to be motion free. This tends to imply that for the data considered here, manual motion correction was effective for all levels of motion corruption seen and could produce parametric estimates with comparable accuracy to data not corrupted by substantial motion. The observation that RCA is the most affected territory agrees with previous research [14].

The proposed uncertainty measure was applied in the comparison of two different motion correction schemes, namely MCAC and MC. With regards to uncertainty in $\sigma_{K_{1}}$, no significant difference between the two strategies was seen. The fact that the two methods produced similar uncertainties could mean that the difference between the two schemes is less important than other factors that contribute to the uncertainty; for example, time sampling, intra-frame motion, scanner noise etc. While MCAC would be the preferred correction scheme as it takes into account the misalignment between the PET series and the attenuation map, our results tend to imply that the MC scheme is sufficient with regards to uncertainty measurement. However, in order to generalize to the wider problem of when correction should be applied, more experiments would be needed, for example exploring the use of deformable registration instead of rigid body translations for attenuation correction.

By measuring the uncertainties, before and after motion correction, we have obtained a quantitative metric that could measure the effectiveness of motion correction. This might be especially useful for high motion cases, where the change was observed to be significant and thus provided reassurance that 


\section{Change in $K_{1}$ after correction for MCAC datasets}

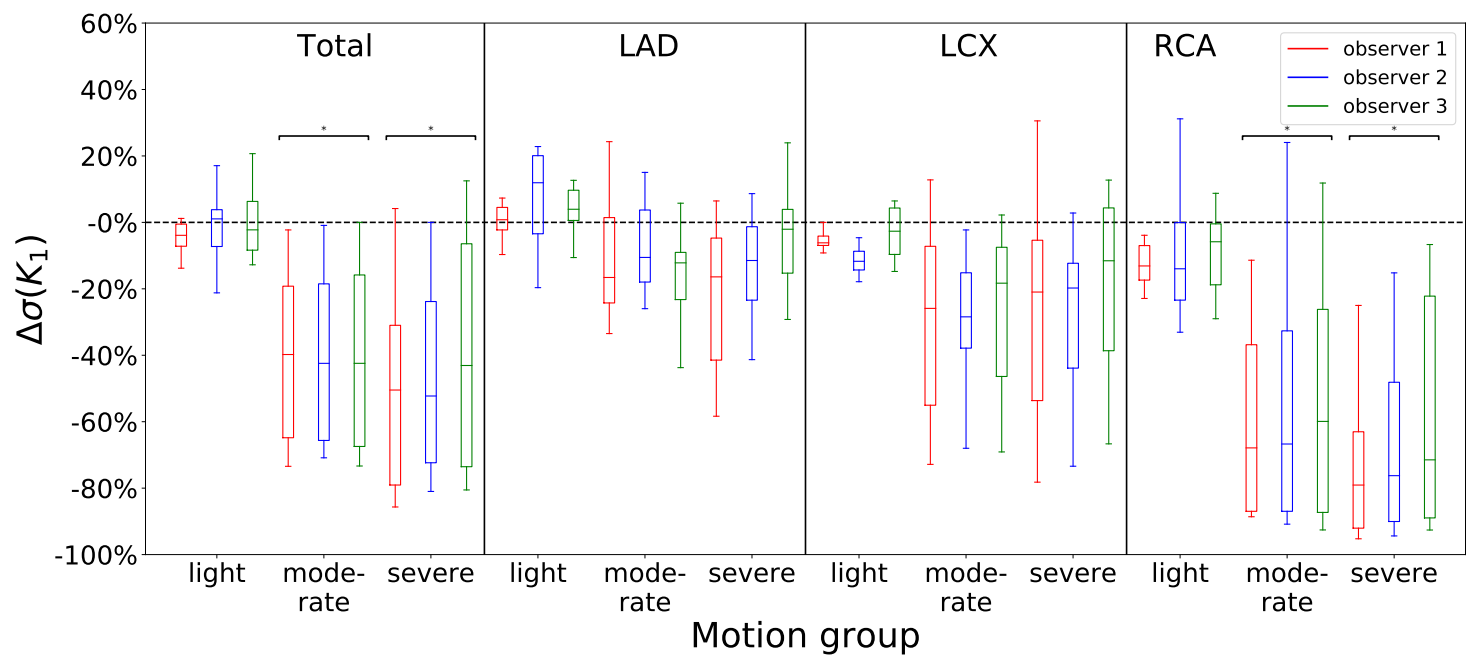

Fig. 8. Change in uncertainty after motion correction for datasets corrected with MCAC. The value $\Delta \sigma_{K_{1}}$ is plotted for the whole myocardium (Total) as well as for each territory. A global decrease can be seen for each motion group. The difference was statistically significant for Total and RCA territory in groups 2 and 3 (p-value $<0.05$ ). The reduction in uncertainty was comparable between observers (median and quartiles are similar).

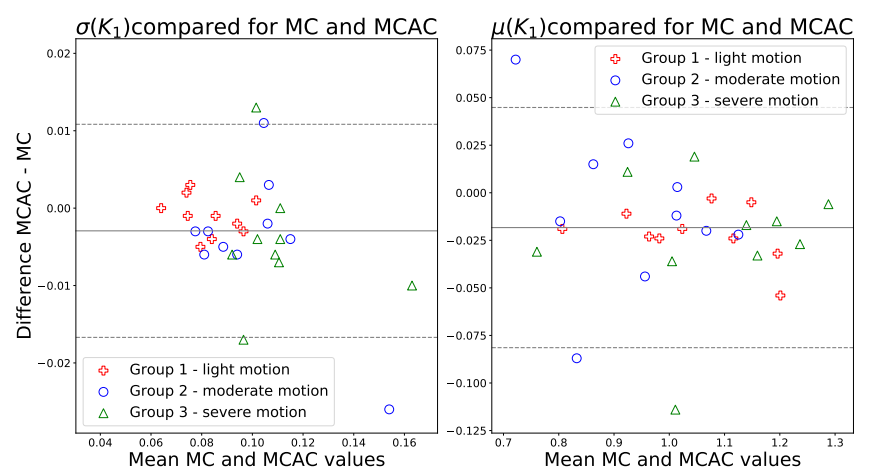

Fig. 9. Bland-Altmann plot of $\sigma_{K_{1}}$ and $\mu_{K_{1}}$ values after motion correction for $\mathrm{MC}$ and MCAC datasets for observer 1. No statistical differences were found between $\mathrm{MC}$ and MCAC correction for each motion group (p-value > $0.05)$

motion correction had been effective. It is also worth noting that while individual observers corrected the datasets to obtain the best visual assessment of motion-free images, the final $\Delta \sigma_{K_{1}}$ was not the same for each observer. In this scenario the uncertainty measure could potentially be used to choose the most effective correction and hence used as an objective way to determine whether motion correction has been of value or to compare different motion correction algorithms. This could be especially useful when automated motion correction is employed allowing the user to objectively determine whether it has been successful.

A potential limitation of this study is the choice of a white noise model for representing the corruption induced by motion in the simulations. While it would be very difficult to describe mathematically the noise induced by motion, it is certainly more complex than a Gaussian distribution as used in our simulated data. However, in a similar manner to the observation in the simulated data where a higher level of noise correlated with higher uncertainty, a similar correlation between higher level of motion and higher uncertainty was observed in the clinical data (Fig. 8 and 10). The fact that the decrease of uncertainty correlates with the independent categorization of the data, implied that our assumption may be valid as far is required for this study.

This study focused primarily on the assessment of motion correction; there are numerous other factors that also have an influence on the reliability of estimated $K_{1}$ values. We observed, for example, that in regions with limited tracer signal in the late frame, which is characteristic of ischemic or infarcted patients, the uncertainty of $K_{1}$ was higher than in healthy tissues. This might influence the evaluation of motion correction using an uncertainty measure, because the change in uncertainty caused by motion may be smaller than for patients without the pathological regions of high uncertainty. Those observations are corroborated by the values of $C V_{K_{1}}$ for light motion datasets before and after motion correction (Fig. 10). The values did not change significantly and were in the range of $C V_{K_{1}}$ for motion-free images, indicating that remaining uncertainty was dominated by other factors and that the influence of motion correction becomes less noticeable. For this data it might thus be possible to give an absolute threshold of K1 uncertainty on which we could base a quality control metric. In this study we have used uncertainty to compare different motion corrections by which to judge whether motion correction is required or has been successful. In principle it might be possible to establish a threshold on the $K_{1}$ uncertainty more generally on which to base decisions regarding motion correction. However, there are various sources of artifact and noise that contribute to uncertainty and these will vary from dataset to dataset, making it challenging to derive such a threshold from this study alone, and requiring further studies with additional datasets.

Although not a limitation in this study, one issue with the 
$\mathrm{CV}\left(K_{1}\right)$ for each motion group calculated before and after motion correction and compared with motion free data
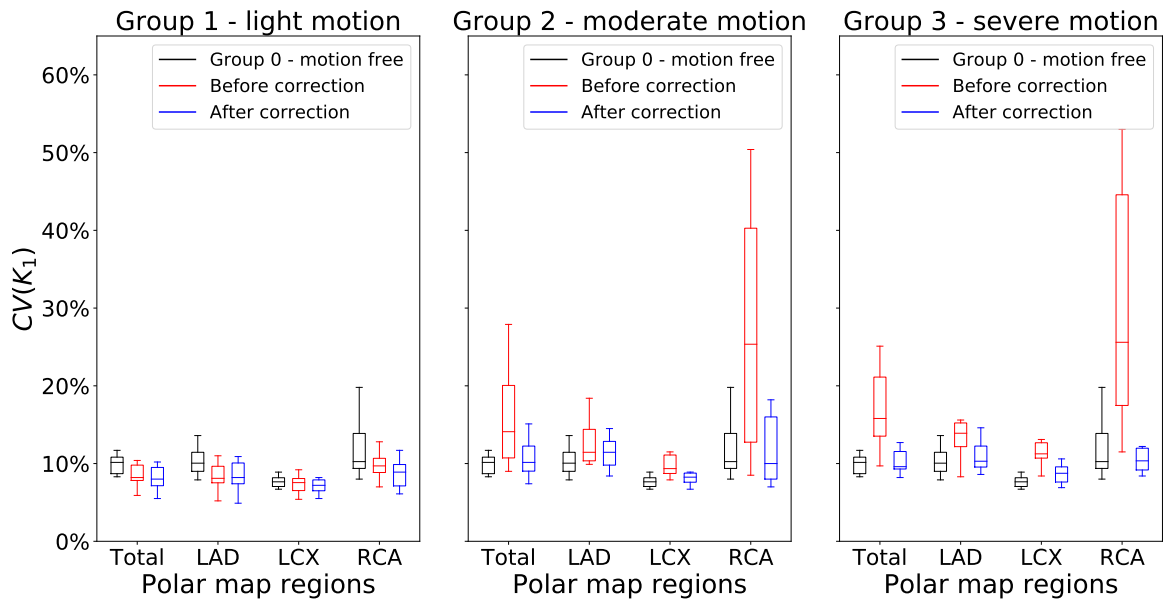

Fig. 10. Comparison of $C V_{K_{1}}$ values before and after motion correction for MCAC datasets for observer 1. The values of $C V_{K_{1}}$, across motion groups after motion correction, were similar to motion free values for each territory.

proposed method in its current implementation is the inability to translate the $K_{1}$ uncertainty into MBF uncertainty. Whilst a reduction of uncertainty in $K_{1}$ will be translated in a reduction in MBF uncertainty (cf. equation 4), it is more challenging to translate $K_{1}$ uncertainty into that of $\mathrm{MBF}$ for any given image, since other parameters play a role into the translation of $K_{1}$ into $\mathrm{MBF}$ as shown in previous studies [5]. However quantitative MBF uncertainty is not a necessity for the purpose of assessing motion correction reliability; and therefore for this limited purpose $K_{1}$ can be used a surrogate for MBF.

\section{CONCLUSION}

By looking at the uncertainty of $K_{1}$, a new method to evaluate the reliability of motion correction of dynamic PET data has been developed. Here a computationally efficient Variational Bayes algorithm has been used for PET kinetic modelling, in order to provide uncertainty estimate of the parameters computed. While the uncertainty is important in itself, with regard to quality control of MBF, this method could potentially be used as a quantitative method to evaluate motion correction algorithms in a systematic manner, which could be evaluated in a future clinical study.

\section{ACKNOWELDGMENTS}

The authors would like to thank Dr. Xiao-Bo Pan for her contributions to the development of this work.

\section{REFERENCES}

[1] V. Dilsizian, S. Bacharach, R. Beanlands, S. Bergmann, D. Delbeke S. Dorbala, R. Gropler, J. Knuuti, H. Schelbert, and M. Travin, "ASNC imaging guidelines/SNMMI procedure standard for Positron Emission Tomography (PET) nuclear cardiology procedures," J. Nucl. Cardiol., vol. 23, pp. 1187-1226, 2016.

[2] M. Ziadi, R. deKemp, K. Williams, A. Guo, J. Renaud, B. Chow, R. Klein, T. Ruddy, M. Aung, L. Garrard, and R. Beanlands, "Does quantification of myocardial flow reserve using rubidium-82 positron emission tomography facilitate detection of multivessel coronary artery disease?" J. Nucl. Cardiol., vol. 19, pp. 671-679, 2011.
[3] P. Camici, R. Gropler, T. Jones, A. L'abbate, A. Maseris, J. Melin P. Merlet, O. Parodi, H. Schelbert, M. Schwaiger, and W. Wijns, "The impact of myocardial blood flow quantitation with PET on the understanding of cardiac diseases," Eur. Heart J., vol. 17, pp. 25-34, 1996.

[4] J. Knuuti, S. Kajander, M. Maki, and H. Ukkonen, "Quantification of myocardial blood flow will reform the detection of CAD," J. Nucl. Cardiol., vol. 16, pp. 497-506, 2009.

[5] J. Moody, V. Murthy, B. Lee, J. Corbett, and E. Ficaro, "Variance Estimation for Myocardial Blood Flow by dynamic PET," IEEE Trans. Med. Imag., vol. 34, pp. 2343-2353, 2015.

[6] J. Moody, B. Lee, J. Corbett, E. Ficaro, and V. Murthy, "Precision and accuracy of clinical quantification of myocardial blood flow by dynamic PET: A technical perspective," J. Nucl. Cardiol., vol. 22, pp. 935-951, 2015.

[7] G. El Fakhri, A. Kardan, A. Sitek, S. Dorbala, N. Abi-Hatem, Y. Lahoud, A. Fischman, M. Coughlan, Y. Tsunehiro, and M. Di Carli, "Reproducibility and Accuracy of Quantitative Myocardial Blood Flow Assessment with 82Rb PET: Comparison with 13N-Ammonia PET," J. Nucl. Med., vol. 50, pp. 1062-1071, 2009.

[8] R. Parkash, R. deKemp, T. Ruddy, A. Kitsikis, R. Hart, L. Beauchesne K. Williams, R. Davies, M. Labinaz, and R. Beanlands, "Potential utility of rubidium 82 PET quantification in patients with 3-vessel coronary artery disease," J. Nucl. Card., vol. 11, pp. 440-449, 2004.

[9] A. Tahari, A. Lee, M. Rajaram, K. Fukushima, M. Lodge, B. Lee, E. Ficaro, S. Nekolla, R. Klein, R. deKemp, R. Wahl, F. Bengel, and P. Bravo, "Absolute Myocardial Flow Quantification with $82 \mathrm{Rb}$ PET/CT: Comparison of Different Software Packages and Methods," $J$. Nucl. Med., vol. 41, 2014.

[10] I. Armstrong, C. Tonge, and P. Arumugam, "Impact of point spread function modeling and time-of-flight on myocardial blood flow and myocardial flow reserve measurements for rubidium-82 cardiac PET," J. Nucl. Cardiol., vol. 21, pp. 467-474, 2014.

[11] B. Lee, J. Moody, R. Weinberg, J. Corbett, E. Picaro, and V. Murthy, "Optimization of temporal sampling for 82-Rubidium PET myocardial blood flow quantification," J. Nucl. Cardiol., vol. 24, pp. 1517-1529, 2017.

[12] C. Hunter, R. Klein, R. Beanlands, and R. deKemp, "Patient motion effects on the quantification of regional myocardial blood flow with dynamic PET imaging," Med. Phys., vol. 43, pp. 1829-1840, 2016.

[13] M. Memmott, C. Tonge, K. Saint, and P. Arumugam, "Impact of pharmacological stress agent on patient motion during rubidium-82 myocardial perfusion PET/CT," J. Nucl. Cardiol., 2017.

[14] B. Lee, J. Moody, A. Poitrasson-Riviere, A. Mevlin, R. Weinberg, R. Corbett, E. Ficaro, and V. Murthy, "Blood pool and tissue phase patient motion effects on 82-Rubidium PET myocardial blood flow quantification," J. Nucl. Cardiol., 2018.

[15] K. Koshino, H. Watabe, J. Enmi, Y. Hirano, T. Zeniya, S. Hasegawa, 
T. Hayashi, S. Miyagawa, Y. Sawa, J. Hatazawa, and H. Iida, "Effects of patient movement on measurements of myocardial blood flow and viability in resting 15O-water PET studies," J. Nucl. Cardiol., 2012.

[16] A. Martinez-Moller, M. Souvatzoglou, N. Navab, M. Schwaiger, and S. Nekolla, "Artifacts from Misaligned CT in Cardiac Perfusion PET/CT Studies: Frequency, Effects, and Potential Solutions," J. Nucl. Med., vol. 48, pp. 188-193, 2007.

[17] M. Ahmed, P. Xiao, and Q. Xie, "New approach for simultaneous respiratory and cardiac motion correction in cardiac PET (NAMCCPET)," Phys. Med. Biol., vol. 60, pp. 7779-7804, 2015.

[18] I. Armstrong, C. Tonge, and P. Arumugam, "Assessing time-of-flight signal-to-noise ratio gains within the myocardium and subsequent reductions in administered activity in cardiac PET studies," J. Nucl. Cardiol., 2017.

[19] H. Fayad, H. Schmidt, C. Wuerslin, and D. Visvikis, "ReconstructionIncorporated Respiratory Motion Correction in Clinical Simultaneous PET/MR Imaging for Oncology Applications," J. Nucl. Med., vol. 56, pp. 884-889, 2015.

[20] M. Omlin and P. Reichert, "A comparison of techniques for the estimation of model prediction uncertainty," Ecological Modelling, vol. 115, pp. 45-59, 1999

[21] G. D'agostini, "Bayesian inference in processing experimental data: principles and basic applications," Rep. Prog. Phys., vol. 66, pp. 13831419, 2003.

[22] J. Bernardo and A. Smith, Bayesian Theory, ser. Wiley Series in Probability and Statistics. John Wiley \& Sons Canada, Limited, 2006.

[23] E. Chib, Siddhartha; Greenberg, "Understanding the MetropolisHastings Algorithm," Am. Statistician, vol. 49, pp. 327-336, 1995.

[24] M. Chappell, A. Groves, and M. Woolrich, "The FMRIB Variational Bayes tutorial," 2008. [Online]. Available: https://users.fmrib.ox.ac.uk/ chappell/papers/TR07MC1.pdf

[25] M. Chappell, A. Groves, B. Whitcher, and M. Woolrich, "Variational Bayesian Inference for a Nonlinear Forward Model," IEEE Trans. Signal Proc., vol. 57, pp. 223-236, 2009.

[26] H. Attias, "A Variational Bayesian Framework for Graphical Models," in In Advances in Neural Information Processing Systems 12. MIT Press, 2000, pp. 209-215.

[27] M. Beal, "Variational algorithms for approximate bayesian inference," Ph.D. dissertation, University College London, 2003.

[28] A. Groves, M. Chappell, and M. Woolrich, "Combined spatial and nonspatial prior for inference on MRI time-series," NeuroImage, vol. 45, pp. 795-809, 2009.

[29] M. Castellaro, G. Rizzo, M. Tonietto, M. Veronese, F. Turkheimer, and M. Chappell, "A Variational Bayesian inference method for parametric imaging of PET data," NeuroImage, vol. 150, pp. 136-149, 2017.

[30] R. Carson, "Tracer Kinetic Modeling in PET," in Positron Emission Tomography: Basic Science, P. Valk, D. Bailey, D. Townsend, and M. Maisey, Eds. Springer, London, 2003, ch. 6, pp. 127-158.

[31] R. Gunn, S. Gunn, and V. Cunningham, "Positron Emission Tomography Compartmental Models," J. Cereb. Blood Flow Metab., vol. 21, pp. 635652, 2001.

[32] M. Lortie, R. Beanlands, K. Yoshinaga, J. Klein, Ran; DaSilva, and R. deKemp, "Quantification of myocardial blood flow with 82Rb dynamic PET imaging," Eur. J. Nucl. Med., vol. 34, pp. 1765-1774, 2007.

[33] R. deKemp, J. Declerck, R. Klein, X.-B. Pan, R. Nakazato, C. Tonge, P. Arumugam, D. Berman, G. Germano, R. Beanlands, and P. Slomka, "Multisoftware Reproducibility Study of Stress and Rest Myocardial Blood Flow Assessed with 3D Dynamic PET/CT and a 1-TissueCompartment Model of 82Rb Kinetics," J. Nucl. Med., vol. 34, 2012.

[34] R. Muzic and B. Christian, "Evaluation of objective functions for estimation of kinetic parameters," Med. Phys., vol. 33, 2006.

[35] K. Chen, S.-C. Huang, and D.-C. Yu, "The effects of measurement errors in the plasma radioactivity curve on parameter estimation in positron emission tomography," Phys. Med. Biol., vol. 36, p. 1183, 1991.

[36] H. M. Hudson and R. S. Larkin, "Accelerated image reconstruction using Ordered Subsets of projection data," IEEE Trans. Med. Imag., vol. 13, pp. 601-609, 1994

[37] D. Tout, C. Tonge, S. Muthu, and P. Arumugam, "Assessment of a protocol for routine simultaneous myocardial blood flow measurement and standard myocardial perfusion imaging with rubidium- 82 on a high count rate positron emission tomography system." Nucl. Med. Commun., vol. 33, pp. 1202-1211, 2012.

[38] B. Bendriem, J. Reed, K. Mccullough, R. Khan, and A. Smith, "The continual innovation of commercial PET/CT solutions in nuclear cardiology: Siemens Healthineers," J. Nucl. Cardiol., pp. 1-12, 2018. 